\title{
Harnack Inequalities and Applications for Multivalued Stochastic Evolution Equations
}

\author{
Shun-Xiang Ouyang* \\ Department of Mathematics, Bielefeld University, D-33501 Bielefeld, Germany \\ School of Math. Sci. \& Lab. Math. Com. Sys.,Beijing Normal University, Beijing 100875, China
}

September 18, 2021

\begin{abstract}
By the method of coupling and Girsanov transformation, Harnack inequalities [F.-Y. Wang, 1997] and strong Feller property are proved for the transition semigroup associated with the multivalued stochastic evolution equation on a Gelfand triple. The concentration property of the invariant measure for the semigroup is investigated. As applications of Harnack inequalities, explicit upper bounds of the $L^{p}$-norm of the density, contractivity, compactness and entropy-cost inequality for the semigroup are also presented.
\end{abstract}

AMS subject Classification (2000): 60H15, 47D07, 37L40

Keywords: Harnack inequality, multivalued stochastic evolution equation, invariant measure, contractivity

\section{Introduction}

Dimension-free Harnack inequality has been extensively studied (see [19, 27]). It is first introduced by Wang [24] for diffusions on Riemannian manifolds with curvature bounded below, then it is considered by Aida and Kawabi $[1,15,16]$ for some infinite dimensional diffusion processes; Röckner and Wang [21] for generalized Mehler semigroup; Arnaudon et al. [3] for diffusions on Riemannian manifolds with curvature unbounded below; Wang [28] for stochastic porous media equations; Da Prato et al. [12] for singular stochastic equations on Hilbert spaces; and the author et al. [20] for Ornstein-Uhnelbeck processes with jumps in infinite dimensional spaces etc..

For the applications of Harnack inequalities, we refer to $[6,21,22,25,26]$ for contractivity properties and functional inequalities; $[1,2,16]$ for short time heat kernel estimates of infinite dimensional diffusions; [12] for regularizing properties; and [4, 13] for heat kernel estimates etc..

*souyang@math.uni-bielefeld.de; Tel.: +49 521 1064760; fax: +49 521 1066455; 
In $[1,15,16,21,24]$ etc. Harnack inequalities were proved via gradient estimate. In [3] the method of coupling and Giranov transformation ([3]) was introduced. This method has been applied in $[12,19,20,28]$ etc.. In this paper, we adopt this method to establish Harnack inequality for multivalued stochastic evolution equations which are generalization of the single valued and multivalued linear case considered respectively in [28].

Multivalued equations attracted the interest of many researchers recently. See Krée [17], Cépa [8, 9, 10], Bensoussan and Rascanu [5], Cépa and Lépingle [11], and Zhang [31] etc. and references therein. We prove Harnack inequalities (see Theorem 4.1) and strong Feller property (see Theorem 5.1) of the invariant measure for the transition semigroup associated with the multivalued stochastic evolution equations in Banach spaces in the framework of [31].

As applications of the Harnack inequalities, we prove the invariant measure is fully supported on the domain of the underlying multivalued maximal monotone operator; and we study the hyperboundedness, ultraboundedness and compactness for the transition semigroup. We also get a log-Harnack inequality and an entropy-cost inequality. We refer to Theorem 5.2 and Theorem 5.3 for details.

Zhang [31, Theorem 5.8] has proved finiteness of the second moment of the invariant measure of the transition semigroup associated with evolution equations. We obtain stronger concentration properties of the invariant measure. See Theorem 3.1.

The organization of this paper follows. We introduce the multivalued stochastic evolution equation in Section 2; study the concentration property of the invariant measure in Section 3; and prove Harnack inequalities in Section 4; then present the applications in Section 5.

\section{Multivalued stochastic evolution equations}

We first recall the definition of multivalued maximal monotone operator. See for instance Brézis [7] for more details.

Denote by $2^{\mathbb{H}}$ for the set of all subsets of $\mathbb{H}$. Let $A: \mathbb{H} \rightarrow 2^{\mathbb{H}}$ be a set-valued operator. Define the domain of $A$ by

$$
D(A)=\{x \in \mathbb{H}: A x \neq \emptyset\} .
$$

The multivalued operator $A$ is characterized by its graph defined by

$$
\operatorname{Gr}(A)=\{(x, y) \in \mathbb{H} \times \mathbb{H}: x \in \mathbb{H}, y \in A x\} .
$$

Definition 2.1. $\quad$ 1. A multivalued operator $A$ on $\mathbb{H}$ is called monotone if

$$
\left\langle x_{1}-y_{1}, x_{2}-y_{2}\right\rangle \geq 0, \quad \text { for all }\left(x_{1}, y_{1}\right),\left(x_{2}, y_{2}\right) \in \operatorname{Gr}(A) \text {. }
$$

2. A monotone operator $A$ is called maximal monotone if it must be $\left(x_{1}, y_{1}\right) \in \operatorname{Gr}(A)$ for any $\left(x_{1}, y_{1}\right) \in \mathbb{H} \times \mathbb{H}$ satisfying

$$
\left\langle x_{1}-x_{2}, y_{1}-y_{2}\right\rangle \geq 0, \quad \text { for all }\left(x_{2}, y_{2}\right) \in \operatorname{Gr}(A) .
$$


That is, $A$ is maximal monotone if its graph $\operatorname{Gr}(A)$ is not contained in the graph of any other monotone operator.

Let $\mathbb{V}$ be a separable and reflexive Banach space which is continuously and densely embedded in a separable Hilbert space $\mathbb{H}$. Then we have an evolution triplet $\left(\mathbb{V}, \mathbb{H}, \mathbb{V}^{*}\right)$ satisfying

$$
\mathbb{V} \subset \mathbb{H}=\mathbb{H}^{*} \subset \mathbb{V}^{*},
$$

where $\mathbb{V}^{*}$ is the dual space of $\mathbb{V}$ and we identify $\mathbb{H}$ with its own dual $\mathbb{H}^{*}$.

Denote by $|\cdot|_{\mathrm{V}},|\cdot|_{\mathbb{H}},|\cdot|_{\mathrm{V}^{*}}$ the norms in $\mathbb{V}, \mathbb{H}$ and $\mathbb{V}^{*}$ respectively; by $\langle\cdot, \cdot\rangle_{\mathbb{H}}$ the inner product in $\mathbb{H}$, and ${ }_{\mathbb{V}}\langle\cdot, \cdot\rangle_{\mathbb{V}^{*}}$ the dual relation between $\mathbb{V}$ and $\mathbb{V}^{*}$. In particular, if $v \in \mathbb{V}$ and $h \in \mathbb{H}$, then

$$
{ }_{\mathrm{v}}\langle v, h\rangle_{\mathbb{V}^{*}}=\langle v, h\rangle_{\mathbb{H}} .
$$

Let $A$ be a multivalued maximal monotone operator on $\mathbb{H}$. We introduce two sets for every $T>0$ :

1. $\mathscr{V}_{T}(\mathbb{H})$ : the set of all $\mathbb{H}$-valued functions of finite variation on $[0, T]$.

2. $\mathscr{A}_{T}$ : the space of all $[u, K]$ such that $u \in C([0, T] ; \overline{D(A)}), K \in \mathscr{V}_{T}(\mathbb{H})$ with $K(0)=0$, and for all $x, y \in C([0, T], \mathbb{H})$ satisfying $(x(t), y(t)) \in \operatorname{Gr}(A)$, the measure

$$
\langle u(t)-x(t), d K(t)-y(t) d t\rangle_{\mathbb{H}} \geq 0 .
$$

Let $W_{t}$ be a cylindrical Wiener process on $\mathbb{H}$ with respect to a filtered probability space $\left(\Omega, \mathscr{F},\left(\mathscr{F}_{t}\right)_{t>0}, \mathbb{P}\right)$. Let $B$ be a single valued operator from $\mathbb{V}$ to $\mathbb{V}^{*}$; and $\sigma$ an operator from $\mathbb{R}_{+} \times \Omega \times \mathbb{H}$ to $\mathbb{H} \otimes \mathbb{H}$. Consider the following multivalued stochastic evolution equation

$$
\left\{\begin{array}{l}
d X_{t} \in-A X_{t} d t+B X_{t} d t+\sigma\left(t, X_{t}\right) d W_{t}, \\
X_{0}=x \in \overline{D(A)} .
\end{array}\right.
$$

Definition 2.2. A pair of $\mathscr{F}_{t}$-adapted random processes $\left(X_{t}, K_{t}\right)$ is called a solution of Equation (1) if

1. $[X(\cdot, \omega), K(\cdot, \omega)] \in \mathscr{A}_{T}$ for almost all $\omega \in \Omega$;

2. For some $q>1, X(\cdot, \omega) \in L^{q}([0, T] ; \mathbb{V})$ for almost all $\omega \in \Omega$;

3. It holds for all $t \in[0, T]$ almost surely

$$
X_{t}=X_{0}-K_{t}+\int_{0}^{t} B X_{s} d s+\int_{0}^{t} \sigma\left(s, X_{s}\right) d W_{s} .
$$

The following theorem on the existence and uniqueness of the equation (1) is due to Zhang [31, Theorem 4.6].

Theorem 2.3. Assume the following conditions. 
(H1) $0 \in D(A)^{o}$, where $D(A)^{\circ}$ denotes the interior of $D(A)$;

(H2) $B$ is hemicontinuous: for every $x, y, z \in \mathbb{V}$,

$$
[0,1] \ni \varepsilon \mapsto \mathrm{V}\langle x, B(y+\varepsilon z)\rangle_{\mathbb{V}^{*}} \text { is continuous; }
$$

(H3) For every $x, y \in \mathbb{V}$,

$$
{ }_{\mathbb{v}}\langle x-y, B x-B y\rangle_{\mathbb{V}^{*}} \leq 0 ;
$$

(H4) There exist $\gamma>0, \omega \in \mathbb{R}$ and $q>1$ such that for every $x, y \in \mathbb{V}$,

$$
{ }_{\mathbb{V}}\langle x-y, B x-B y\rangle_{\mathbb{V}^{*}} \leq-\gamma|x-y|_{\mathrm{V}}^{q}+\omega|x-y|_{\mathrm{H}}^{2} ;
$$

(H5) There exists a $C>0$ such that for every $x \in \mathbb{V}$,

$$
|B x|_{\mathbb{V}^{*}} \leq C\left(1+|x|_{\mathrm{V}}^{q-1}\right),
$$

where $q$ is the same as in (2);

(H6) Let $\mathcal{M}$ be the set of all progressively measurable sets with respect to $\mathscr{F}_{t}$. Assume $\sigma$ is $\mathcal{M} \times \mathcal{B}(\mathbb{H}) / \mathcal{B}(\mathbb{H} \otimes \mathbb{H})$ measurable and there exists a positive constant $C_{\sigma}$ such that for all $(t, \omega) \in \mathbb{R}_{+} \times \Omega$ and $x, y \in \mathbb{H}$,

$$
\begin{aligned}
& \|\sigma(t, \omega, x)-\sigma(t, \omega, y)\|_{\mathrm{H} \otimes \mathrm{H}} \leq C_{\sigma}|x-y|_{\mathrm{H}}, \\
& \|\sigma(t, \omega, x)\|_{\mathrm{H} \otimes \mathbb{H}} \leq C_{\sigma}\left(1+|x|_{\mathrm{H}}\right) .
\end{aligned}
$$

Then there exists a unique solution to Equation (1) in the sense of Definition 2.2.

The following proposition will play an important role. See Zhang [31, Proposition 3.3] for a proof.

Proposition 2.4. Let $[u, K],[\tilde{u}, \widetilde{K}] \in \mathscr{A}_{T}$. Then the measure

$$
\langle u(t)-\tilde{u}(t), d K(t)-d \widetilde{K}(t)\rangle_{\mathbb{H}} \geq 0, \quad t \in[0, T] .
$$

\section{Concentration of invariant measures}

Suppose that Conditions (H1)-(H6) hold. By Theorem 2.3, Equation (1) has a unique solution $X_{t}$. Define

$$
P_{t} f(x)=\mathbb{E}_{\mathbb{P}} f\left(X_{t}\right)
$$

for every $t \in[0, T], f \in \mathscr{B}_{b}(\overline{D(A)})$. Let $\sigma$ be deterministic and time independent. Then $P_{t}$ is a Markov semigroup (see [31, Theorem 5.5]).

Zhang [31, Theorem 5.8] has studied the the existence, uniqueness of the invariant measure associated with $P_{t}$. He also proved that the invariant measure $\mu$ satisfies $\mu\left(|x|_{\mathrm{H}}^{2}\right)<\infty$. We prove a stronger concentration properties. 
Theorem 3.1. Assume that (H1)-(H6) hold with $q \geq 2$. Let $\sigma$ be deterministic and independent of time. Assume further that $\mathbb{V}$ is compactly embedded in $\mathbb{H}$. If $q=2$, then suppose in addition that $\sigma$ is uniformly bounded and $\lambda \omega<\gamma$, where $\lambda$ is the constant such that $|\cdot|_{\mathbb{H}} \leq \lambda|\cdot|_{\mathbb{V}}$. Then there exist an invariant measure associated with $P_{t}$ in the sense that

$$
\int_{\overline{D(A)}} P_{t} f(x) \mu(d x)=\int_{\overline{D(A)}} f(x) \mu(d x), \quad f \in \mathscr{B}_{b}(\overline{D(A)}) .
$$

Moreover,

$$
\int_{\overline{D(A)}}|x|_{\mathbb{V}}^{q} \mu(d x)<\infty .
$$

If $\sigma$ is uniformly bounded, then for every $q \geq 2$, there exist some $\theta>0$ such that

$$
\int_{\overline{D(A)}} \mathrm{e}^{\theta|x|_{\mathrm{H}}^{q}} \mu(d x)<\infty .
$$

Proof. (1) The existence of the invariant measures has been proved in [31, Theorem 5.8 (i)] for the case $q>2$. The extension to the case $q=2$ is not hard. We skip the proof here since the main technique can be found below.

(2) From (H3) we know for all $x \in \mathbb{V}$,

$$
\mathbb{V}\langle x, B x\rangle_{\mathbb{V}^{*}} \leq-\gamma|x|_{\mathbb{V}}^{q}+\omega|x|_{\mathbb{H}}^{2}+\mathbb{V}\langle x, B 0\rangle_{\mathbb{V}^{*}} .
$$

If $q=2$, then

$$
\omega|x|_{\mathbb{H}}^{2} \leq \lambda \omega|x|_{\mathbb{V}}^{2}<\gamma|x|_{\mathbb{V}}^{2}
$$

If $q>2$, then by Young's inequality,

$$
\omega|x|_{\mathrm{H}}^{2} \leq \lambda \omega|x|_{\mathbb{V}}^{2} \leq \frac{2 \varepsilon^{q}}{q}|x|_{\mathbb{V}}^{q}+\frac{(\lambda \omega)^{p^{\prime}}}{p^{\prime} \varepsilon^{p^{\prime}}},
$$

hold for every $\varepsilon>0$, where $p^{\prime}$ satisfying $1 / p^{\prime}+2 / q=1$.

Use the estimate (6) and (7) in (5) for $q=2$ and $q>2$ (by taking $\varepsilon$ small enough in this case) respectively, we know there are constants $C_{1}, \gamma^{\prime}>0$ such that

$$
\mathbb{V}\langle x, B x\rangle_{\mathbb{V}^{*}} \leq C_{1}-2 \gamma^{\prime}|x|_{\mathbb{V}}^{q}+\mathbb{V}\langle x, B 0\rangle_{\mathbb{V}^{*}}
$$

By Young's inequality again, we know for any $\tilde{\varepsilon}>0$,

$$
{ }_{\mathbb{V}}\langle x, B 0\rangle_{\mathbb{V}^{*}} \leq|x|_{\mathbb{V}} \cdot|B 0|_{\mathbb{V}^{*}} \leq \frac{\tilde{\varepsilon}^{q}}{q}|x|_{\mathbb{V}}^{q}+\frac{1}{p \tilde{\varepsilon}^{p}}|B 0|_{\mathbb{V}^{*}}^{p},
$$

where $p=\frac{q}{q-1}$.

Therefore, we deduce from (8) and (9) by taking $\tilde{\varepsilon}$ small enough to get

$$
{ }_{\mathbb{V}}\langle x, B x\rangle_{\mathbb{V}^{*}} \leq C_{2}-\gamma^{\prime}|x|_{\mathbb{V}}^{q}
$$

for some constant $C_{2}, \gamma^{\prime}>0$. 
Now we fix a $y \in A 0$. Let $\left(X_{t}, Y_{t}\right)$ be the solution to the multivalued stochastic evolution equation (1). By definition, we have

$$
\left\langle X_{t}-0, d K(t)-y d t\right\rangle \geq 0,
$$

By Itô's formula, using (10) and (11) and Young's inequality again, we obtain

$$
\begin{aligned}
& \frac{1}{2} d\left|X_{t}\right|_{\mathbb{H}}^{2} \\
\leq & -{ }_{\mathbb{V}}\left\langle X_{t}, B X_{t}\right\rangle_{\mathbb{V}^{*}} d t-\left\langle X_{t}, d K_{t}\right\rangle_{\mathbb{H}} d t+\frac{1}{2}\|\sigma\|_{\mathrm{H} \otimes \mathbb{H}}^{2} d t+\left\langle X_{t}, \sigma d W_{t}\right\rangle \\
\leq & \left(C_{3}-\gamma^{\prime}\left|X_{t}\right|_{\mathbb{V}}^{q}\right) d t+|y|_{\mathbb{H}} \cdot\left|X_{t}\right|_{\mathbb{H}} d t+\left\langle X_{t}, \sigma d W_{t}\right\rangle \\
\leq & \left(C_{4}-\frac{\gamma^{\prime}}{2}\left|X_{t}\right|_{\mathbb{V}}^{q}\right) d t+\left\langle X_{t}, \sigma d W_{t}\right\rangle,
\end{aligned}
$$

where $C_{3}, C_{4}>0$ are some constants.

In the calculation of (12) we also used Young's inequality to get control from $\|\sigma(x)\|_{\mathbb{H} \times \mathbb{H}} \leq$ $C_{\sigma}\left(1+|x|_{\mathrm{H}}\right)$ if $q$ is strictly greater than 2. If $q=2$, we use the assumption that $\sigma$ uniformly bounded.

Therefore, by (12), we get

$$
\int_{0}^{1} \frac{\gamma^{\prime}}{2} \mathbb{E}^{x}\left|X_{s}\right|_{\mathrm{V}}^{q} d s \leq C_{4}+\frac{1}{2}\left(|x|_{\mathrm{H}}^{2}-\mathbb{E}^{x}\left|X_{1}\right|_{\mathrm{H}}^{2}\right) .
$$

Consequently we have

$$
\int_{0}^{1} P_{s}|\cdot|_{\mathrm{V}}^{q}(x) d s \leq \frac{1}{\gamma^{\prime}}\left(2 C_{4}+|x|_{\mathrm{H}}^{2}\right) .
$$

Hence we obtain $\mu\left(|\cdot|_{\mathrm{V}}^{q}\right)<\infty$. This proves (3).

(3) For every $\theta>0$, by (12) we have

$$
\begin{aligned}
& d \mathrm{e}^{\theta\left|X_{t}\right|_{\mathrm{H}}^{q}} \\
= & \frac{1}{2} \theta q\left|X_{t}\right|_{\mathrm{H}}^{q-2} \mathrm{e}^{\theta\left|X_{t}\right|_{\mathrm{H}}^{q}} d\left|X_{t}\right|_{\mathrm{H}}^{2} \\
& \quad+\frac{1}{2}\left(\frac{1}{2} \theta q \mathrm{e}^{\theta\left|X_{t}\right|_{\mathrm{H}}^{q}}\right)\left(\frac{1}{2} \theta q\left|X_{t}\right|_{\mathrm{H}}^{2(q-2)}+\frac{q-2}{2}\left|X_{t}\right|_{\mathrm{H}}^{q-4}\right) d\left\langle\left|X_{t}\right|_{\mathrm{H}}^{2},\left|X_{t}\right|_{\mathrm{H}}^{2}\right\rangle \\
= & \frac{1}{2} \theta q\left|X_{t}\right|_{\mathrm{H}}^{q-2} \mathrm{e}^{\theta\left|X_{t}\right|_{\mathrm{H}}^{q}}\left(d\left|X_{t}\right|_{\mathrm{H}}^{2}+2 \theta q|\sigma|_{\mathrm{H} \otimes \mathrm{H}}^{2}\left|X_{t}\right|_{\mathrm{H}}^{q} d t+(q-2)|\sigma|_{\mathrm{H} \otimes \mathrm{H}}^{2} d t\right) \\
\leq & \frac{1}{2} \theta q\left|X_{t}\right|_{\mathrm{H}}^{q-2} \mathrm{e}^{\theta\left|X_{t}\right|_{\mathrm{H}}^{q}}\left(C_{5}-\gamma^{\prime}\left|X_{t}\right|_{\mathrm{V}}^{q}+2 \theta q|\sigma|_{\mathrm{H} \otimes \mathrm{H}}^{2}\left|X_{t}\right|_{\mathrm{H}}^{q}\right) d t+d M_{t}
\end{aligned}
$$

for some constant $C_{5}>0$ and some local martingale $M_{t}$.

Since $|\cdot|_{\mathbb{H}} \leq \lambda|\cdot|_{\mathrm{V}}$, for small enough $\theta$, we have

$$
d \mathrm{e}^{\theta\left|X_{t}\right|_{\mathrm{H}}^{q}} \leq \frac{1}{2} \theta q\left|X_{t}\right|_{\mathrm{H}}^{q-2} \mathrm{e}^{\theta\left|X_{t}\right|_{\mathrm{H}}^{q}}\left(C_{5}-\frac{\gamma^{\prime}}{2}\left|X_{t}\right|_{\mathrm{V}}^{q}\right) d t+d M_{t} .
$$


Let us focus at the drift of the right hand of (15). By the fact $|\cdot|_{\mathbb{H}} \leq \lambda|\cdot|_{\text {V }}$ and Young's inequality,

$$
\begin{aligned}
\frac{1}{2} \theta q\left|X_{t}\right|_{\mathbb{H}}^{q-2}\left(C_{5}-\frac{\gamma^{\prime}}{2}\left|X_{t}\right|_{\mathbb{V}}^{q}\right) & \leq \frac{1}{2} \theta q C_{5}\left|X_{t}\right|_{\mathbb{H}}^{q-2}-\frac{1}{2} \theta q \cdot \frac{\gamma^{\prime}}{2} \lambda^{-q}\left|X_{t}\right|_{\mathbb{H}}^{q} \cdot\left|X_{t}\right|_{\mathbb{H}}^{q-2} \\
& \leq C_{6}-\gamma^{\prime \prime}\left|X_{t}\right|_{\mathbb{H}}^{2(q-1)}
\end{aligned}
$$

for some constant $C_{6}, \gamma^{\prime \prime}>0$.

Now let

$$
G=\left\{\left|X_{t}\right|_{\mathbb{H}}^{2(q-1)} \geq 1+\frac{C_{6}}{\gamma^{\prime \prime}}\right\}
$$

Note that on $G^{c}$, both $\left|X_{t}\right|_{\mathbb{H}}^{2(q-1)}$ and $\mathrm{e}^{\theta\left|X_{t}\right|_{\mathrm{H}}^{q}}$ are bounded. Therefore

$$
\begin{aligned}
& \left(C_{6}-\gamma^{\prime \prime}\left|X_{t}\right|_{\mathrm{H}}^{2(q-1)}\right) \mathrm{e}^{\theta\left|X_{t}\right|_{\mathrm{H}}^{q}} \\
= & -\gamma^{\prime \prime}\left(\left|X_{t}\right|_{\mathrm{H}}^{2(q-1)}-\frac{C_{6}}{\gamma^{\prime \prime}}\right) \mathrm{e}^{\theta\left|X_{t}\right|_{\mathrm{H}}^{q}} \\
\leq & -\gamma^{\prime \prime} \mathrm{e}^{\theta\left|X_{t}\right|_{\mathrm{H}}^{q}} \mathbb{1}_{G}-\gamma^{\prime \prime}\left(\left|X_{t}\right|_{\mathrm{H}}^{2(q-1)}-\frac{C_{6}}{\gamma^{\prime \prime}}\right) \mathrm{e}^{\theta\left|X_{t}\right|_{\mathrm{H}}^{q}} \mathbb{1}_{G^{c}} \\
\leq & -\gamma^{\prime \prime} \mathrm{e}^{\theta\left|X_{t}\right|_{\mathrm{H}}^{q}}+\gamma^{\prime \prime} \mathrm{e}^{\theta\left|X_{t}\right|_{\mathrm{H}}^{q}} \mathbb{1}_{G^{c}}-\gamma^{\prime \prime}\left(\left|X_{t}\right|_{\mathrm{H}}^{2(q-1)}-\frac{C_{6}}{\gamma^{\prime \prime}}\right) \mathrm{e}^{\theta\left|X_{t}\right|_{\mathrm{H}}^{q}} \mathbb{1}_{G^{c}} \\
\leq & C_{7}-\gamma^{\prime \prime} \mathrm{e}^{\theta\left|X_{t}\right|_{\mathrm{H}}^{q}}
\end{aligned}
$$

for some constant $C_{7}>0$.

Hence from (16) and (17), we can get an estimate of the drift of the right hand side of (15). That is,

$$
d \mathrm{e}^{\theta\left|X_{t}\right|_{\mathrm{H}}^{q}} \leq\left(C_{7}-\gamma^{\prime \prime} \mathrm{e}^{\theta\left|X_{t}\right|_{\mathrm{H}}^{q}}\right) d t+d M_{t}
$$

By integrating the inequality (18) from 0 to $n$, we get

$$
\mathrm{e}^{\theta\left|X_{n}\right|_{\mathrm{H}}^{q}} \leq \mathrm{e}^{\theta\left|X_{0}\right|_{\mathrm{H}}^{q}}+C_{7} n-\gamma^{\prime \prime} \int_{0}^{n} \mathrm{e}^{\theta\left|X_{s}\right|_{\mathrm{H}}^{q}} d s+M_{n} .
$$

Then we take expectation for both side of (19) with respect to $\mathbb{P}^{0}$, we get

$$
\mathbb{E} \mathrm{e}^{\theta\left|X_{n}\right|_{\mathrm{H}}^{q}} \leq 1+C_{7} n-\gamma^{\prime \prime} \int_{0}^{n} \delta_{0} P_{s} \mathrm{e}^{\theta|\cdot|_{\mathrm{H}}^{q}} d s
$$

It follows that

$$
\mu_{n}\left(\mathrm{e}^{\left.\theta|\cdot|\right|_{\mathrm{H}} ^{q}}\right) \leq \frac{C_{7}}{\gamma^{\prime \prime}}+\frac{1}{n \gamma^{\prime \prime}}, \quad n \geq 1
$$

where

$$
\mu_{n}=\frac{1}{n} \int_{0}^{n} \delta_{0} P_{s} d s, \quad n \geq 1
$$

Note that $\mu$ is the weak limit of $\mu_{n}$ (refer to the proof of $[31,5.8]$ ), we can deduce from (21) to get $\mu\left(\mathrm{e}^{\theta|\cdot|_{\mathrm{H}}^{q}}\right)<\infty$. This proves (4). 


\section{$4 \quad$ Harnack inequalities}

In the following we assume conditions (H1)-(H5) in Theorem 2.3 and instead of (H6) we suppose that

$\left(\mathbf{H 6}^{\prime}\right) \sigma:[0, \infty) \times \Omega \rightarrow \mathbb{H} \otimes \mathbb{H}$ be a nondegenerate Hilbert-Schmidt operator uniformly bounded in time $t \in[0, \infty)$ and $\omega \in \Omega$.

For every $x \in \mathbb{H}$, define

$$
|x|_{\sigma_{t}}= \begin{cases}|y|_{\mathbb{H}} & \text { if } x=\sigma_{t} y \text { for some } y \in \mathbb{H} \\ \infty, & \text { otherwise. }\end{cases}
$$

The distance associated with $|\cdot|_{\sigma_{t}}$ is called the intrinsic distance induced by $\sigma_{t}$.

We are going to prove the following Harnack inequality for the semigroup $P_{t}$ associated with the solution process of Equation (1).

Theorem 4.1. Assume (H1)-(H5) and $\left(H 6^{\prime}\right)$. Suppose that there exists some nonnegative constant $r \geq q-4$, and some strictly positive continuous function $\zeta_{t}$ on $[0, \infty)$ such that

$$
\zeta_{t}^{2}|x|_{\sigma_{t}}^{2+r} \cdot|x|_{\mathbb{H}}^{q-2-r} \leq|x|_{\mathbb{V}}^{q}, \quad \text { for all } x \in \mathbb{V}, t \geq 0
$$

holds on $\Omega$. Then for every $T>0, \alpha>1, x, y \in \overline{D(A)}$ and $f \in \mathscr{C}_{b}^{+}(\overline{D(A)})$, the following inequality holds

$$
\left(P_{T} f\right)^{\alpha}(x) \leq \exp \left(\frac{\alpha}{2(\alpha-1)} \Theta_{T}|x-y|_{\mathbb{H}}^{\frac{2(4+r-q)}{2+r}}\right) P_{T} f^{\alpha}(y),
$$

where

$$
\Theta_{T}=4 \delta^{-\frac{2(3+r)}{2+r}} \gamma^{-\frac{2}{2+r}} \frac{\left(\int_{0}^{T} \zeta_{t}^{2} \mathrm{e}^{-\delta \omega t} d t\right)^{\frac{r}{2+r}}}{\left(\int_{0}^{T} \zeta_{t} \mathrm{e}^{-\delta \omega t} d t\right)^{2}}
$$

with

$$
\delta=1-\frac{q}{4+r} .
$$

Assume the diffusion coefficient $\sigma$ is independent of $(t, \omega)$ and the function $\zeta_{t}$ in $(22)$ is taken as constant $\zeta$. Then (23) holds with $\Theta_{T}$ replaced by

$$
\widetilde{\Theta}_{T}=4 \delta^{-1} \gamma^{-\frac{2}{2+r}} \zeta^{-\frac{4}{2+r}}\left[\omega^{-1}\left(1-\mathrm{e}^{-\delta \omega T}\right)\right]^{-\frac{4+r}{2+r}}
$$

Proof. The proof is divided into six steps. We outline the main procedure of the proof of (23) in the first step and then realize the idea in the next four steps. The simplification from (24) to (26) is obtained in the last step.

(1) Main Idea. 
Consider the following coupled multivalued stochastic evolution equation

$$
\left\{\begin{array}{l}
d X_{t} \in-A X_{t} d t+B X_{t} d t+\sigma(t) d W_{t}-U_{t} d t \\
d Y_{t} \in-A Y_{t} d t+B Y_{t} d t+\sigma(t) d W_{t}
\end{array}\right.
$$

with initial conditions $X_{0}=x \in \overline{D(A)}, Y_{0}=y \in \overline{D(A)}$, and the drift $U_{t}$ in (27a) is of the following form

$$
U_{t}=\frac{\eta_{t}\left(X_{t}-Y_{t}\right)}{\left|X_{t}-Y_{t}\right|_{\mathrm{H}}^{\delta}} \mathbb{1}_{\{t<\tau\}}
$$

where the stopping time $\tau$ in (28) is the coupling time of $X_{t}$ and $Y_{t}$ defined by

$$
\tau=\inf \left\{t \geq 0: X_{t}=Y_{t}\right\},
$$

the power $\delta$ in $(28)$ is a constant in $(0,1)$ (see $(25))$ and $\eta_{t}$ is a deterministic function on $[0, \infty)$. Both $\delta$ and $\eta_{t}$ in (28) will be specified later such that the following two crucial conditions

$$
X_{T}=Y_{T} \quad \text { a.s. }
$$

and

$$
\mathbb{E}_{\mathbb{P}} \exp \left(\int_{0}^{T} \frac{\eta_{t}^{2}}{2} \frac{\left|X_{t}-Y_{t}\right|_{\sigma_{t}}^{2}}{\left|X_{t}-Y_{t}\right|_{\mathbb{H}}^{2 \delta}} \mathbb{1}_{\{t<\tau\}} d t\right)<\infty
$$

are satisfied.

Let $N_{t}=\int_{0}^{t}\left\langle\sigma_{s}^{-1} U_{s}, d W_{s}\right\rangle$. By (30) we know

$$
R_{t}=\exp \left(N_{t}-\frac{1}{2}[N]_{t}\right), \quad t \in[0, T]
$$

is a martingale on $\left(\Omega, \mathscr{F}_{T},\left(\mathscr{F}_{t}\right)_{0 \leq t \leq T}, \mathbb{P}\right)$. Then we can define a new probability measure $\mathbb{Q}$ on $\left(\Omega, \mathscr{F}_{T}\right)$ by setting $\left.\mathbb{Q}\right|_{\mathscr{F}_{T}}=R_{T} \mathbb{P}$. By Girsanov's theorem,

$$
\widetilde{W}_{t}:=W_{t}-\int_{0}^{t} \sigma_{s}^{-1} U_{s} d s
$$

is still a cylindrical Wiener process on $\left(\Omega, \mathscr{F}_{T},\left(\mathscr{F}_{t}\right)_{0 \leq t \leq T}, \mathbb{Q}\right)$. Hence Equation (27a) can be rewritten in the following way

$$
d X_{t} \in-A X_{t} d t+B X_{t} d t+\sigma(t) d \widetilde{W}_{t}
$$

with initial condition $X_{0}=x$.

By the uniqueness of the solution, the transition law of $\left(X_{t}\right)_{t \in[0, T]}$ under $\mathbb{Q}$ is the same with the transition law of $\left(Y_{t}\right)_{t \in[0, T]}$ under $\mathbb{P}$. So by the fact $(29)$ which will be verified, we have

$$
P_{T} f(x)=\mathbb{E}_{\mathbb{Q}} f\left(X_{T}\right)=\mathbb{E}_{\mathbb{Q}} f\left(Y_{T}\right)=\mathbb{E}_{\mathbb{P}} R f\left(Y_{T}\right) .
$$

Note that we also have $P_{T} f(y)=\mathbb{E}_{\mathbb{P}} f\left(Y_{T}\right)$, therefore by applying Hölder's inequality to (31), we get

$$
\left(P_{T} f\right)^{\alpha}(x) \leq\left(\mathbb{E}_{\mathbb{P}} R_{T}^{\alpha /(\alpha-1)}\right)^{\alpha-1} P_{T} f^{\alpha}(y) .
$$


Then the proof is completed by estimating $\mathbb{E}_{\mathbb{P}} R_{T}^{\alpha /(\alpha-1)}$.

(2) Existence of the solution of the coupled equation (27).

Note that the function

$$
(u, v) \mapsto \frac{u-v}{|u-v|_{H}^{\delta}}
$$

satisfies the monotone condition off the diagonal (see [28, Appendix A]).

By applying Theorem 2.3 we see the coupled equation (27) has a solution up to the coupling time $\tau$. So there exists continuous processes $(X, K) \in \mathscr{A}_{T \wedge \tau}$ and $(Y, \widetilde{K}) \in \mathscr{A}_{T \wedge \tau}$ such that for all $t<\tau$,

$$
\left\{\begin{array}{l}
X_{t}=x-K_{t}+\int_{0}^{t} B X_{s} d s+\int_{0}^{t} \sigma(s) d W_{s}-\int_{0}^{t} U_{s} d s \\
Y_{t}=y-\widetilde{K}_{t}+\int_{0}^{t} B Y_{s} d s+\int_{0}^{t} \sigma(s) d W_{s}
\end{array}\right.
$$

On the other hand, it is obvious that the solution of Equation (27b) (or equivalently, Equation $(33 \mathrm{~b})$ ) can be extended to be a solution for all time $t \in[0, T]$. Let $\left(Y_{t}, \widetilde{K}\right)_{t \geq 0}$ solves Equation (27b). Now we get solution of Equation (27a) (or (33a)) by defining $X_{t}=Y_{t}, K_{t}=\widetilde{K}_{t}$ for all $t \geq \tau$.

(3) Verify (29).

Apply Itô's formula (see e.g. [18]) or Zhang [31, Theorem A.1] etc.) to $\sqrt{\left|X_{t}-Y_{t}\right|_{\mathrm{H}}^{2}+\varepsilon}$ and then let $\varepsilon \downarrow 0$, by using condition (H4) we have for $t<\tau$

$$
\begin{aligned}
d\left|X_{t}-Y_{t}\right|_{\mathbb{H}}^{2} \leq & -\left\langle X_{t}-Y_{t}, d K_{t}-d \widetilde{K}_{t}\right\rangle_{\mathbb{H}} d t \\
& +\left(-\gamma\left|X_{t}-Y_{t}\right|_{\mathbb{V}}^{q}+\omega\left|X_{t}-Y_{t}\right|_{\mathbb{H}}^{2}-\eta_{t}\left|X_{t}-Y_{t}\right|_{\mathbb{H}}^{2-\delta}\right) d t .
\end{aligned}
$$

By Proposition 2.4, for all $t<\tau$ we have

$$
d\left|X_{t}-Y_{t}\right|_{\mathbb{H}}^{2} \leq\left(-\gamma\left|X_{t}-Y_{t}\right|_{\mathbb{V}}^{q}+\omega\left|X_{t}-Y_{t}\right|_{\mathbb{H}}^{2}-\eta_{t}\left|X_{t}-Y_{t}\right|_{\mathbb{H}}^{2-\delta}\right) d t
$$

Then

$$
d\left(\left|X_{t}-Y_{t}\right|_{\mathbb{H}}^{2} \mathrm{e}^{-\omega t}\right) \leq-\mathrm{e}^{-\omega t}\left(\gamma\left|X_{t}-Y_{t}\right|_{\mathbb{V}}^{q}+\eta_{t}\left|X_{t}-Y_{t}\right|_{\mathbb{H}}^{2-\delta}\right) d t .
$$

Hence by (34) we get

$$
\begin{aligned}
d\left(\left|X_{t}-Y_{t}\right|_{\mathbb{H}}^{2} \mathrm{e}^{-\omega t}\right)^{\delta / 2} & \leq \frac{\delta}{2}\left(\left|X_{t}-Y_{t}\right|_{\mathbb{H}}^{2} \mathrm{e}^{-\omega t}\right)^{\delta / 2-1} \cdot\left(-\mathrm{e}^{-\omega t} \eta_{t}\left|X_{t}-Y_{t}\right|_{\mathbb{H}}^{2-\delta}\right) d t \\
& =-\frac{\delta}{2} \mathrm{e}^{-\frac{\delta}{2} \omega t} \eta_{t} d t
\end{aligned}
$$

We take

$$
\eta_{t}=\vartheta_{T} \zeta_{t} \mathrm{e}^{-\frac{\delta}{2} \omega t}
$$

with

$$
\vartheta_{T}=\frac{2 \delta^{-1}|x-y|_{\mathbb{H}}^{\delta}}{\int_{0}^{T} \zeta_{t} \mathrm{e}^{-\delta \omega t} d t}
$$


It must be $T \geq \tau$ and hence $X_{T}=Y_{T}$. Otherwise, if $T<\tau$, by taking integral from 0 to $T$ for both sides of the inequality (35), we can obtain

$$
\left|X_{T}-Y_{T}\right|_{\mathbb{H}}^{\delta} \mathrm{e}^{-\frac{\delta}{2} \omega T} \leq|x-y|_{\mathbb{H}}^{\delta}-\frac{\delta}{2} \int_{0}^{T} \mathrm{e}^{-\frac{\delta}{2} \omega t} \eta_{t} d t .
$$

By (36) the right hand side of (37) equals to 0 . So we can conclude $X_{T}=Y_{T}$ from (37). But this is contradict with the assumption that $T<\tau$.

(4) Verify (30).

From (34) and the assumption (22) we can get for all $t \leq \tau$

$$
\begin{aligned}
d\left(\left|X_{t}-Y_{t}\right|_{\mathbb{H}}^{2} \mathrm{e}^{-\omega t}\right)^{\delta} & =\delta\left(\left|X_{t}-Y_{t}\right|_{\mathbb{H}}^{2} \mathrm{e}^{-\omega t}\right)^{\delta-1} d\left(\left|X_{t}-Y_{t}\right|_{\mathbb{H}}^{2} \mathrm{e}^{-\omega t}\right) \\
& \leq-\delta \gamma \mathrm{e}^{-\delta \omega t}\left|X_{t}-Y_{t}\right|_{\mathbb{H}}^{2(\delta-1)} \cdot\left|X_{t}-Y_{t}\right|_{\mathbb{V}}^{q} d t \\
& \leq-\delta \gamma \zeta_{t}^{2} \mathrm{e}^{-\delta \omega t} \frac{\left|X_{t}-Y_{t}\right|_{\sigma_{t}}^{2+r}}{\left|X_{t}-Y_{t}\right|_{\mathbb{H}}^{2+r-2(\delta-1)-q}} d t
\end{aligned}
$$

Let $\delta$ be defined as in (25). Then from (38) we get

$$
d\left(\left|X_{t}-Y_{t}\right|_{\mathbb{H}}^{2} \mathrm{e}^{-\omega t}\right)^{\delta} \leq-\delta \gamma \zeta_{t}^{2} \mathrm{e}^{-\delta \omega t} \frac{\left|X_{t}-Y_{t}\right|_{\sigma_{t}}^{2+r}}{\left|X_{t}-Y_{t}\right|_{\mathbb{H}}^{\delta(2+r)}} d t
$$

According to (36), we have $\zeta_{t}^{2}=\frac{\eta_{t}^{2}}{\vartheta_{T}^{2}} \mathrm{e}^{-\delta \omega t}$. Now by integrating both sides of the inequality (39) from 0 to $T$, we get (note that $X_{T}=Y_{T}$ )

$$
\frac{\delta \gamma}{\vartheta_{T}^{2}} \int_{0}^{T} \frac{\eta_{t}^{2}\left|X_{t}-Y_{t}\right|_{\sigma_{t}}^{2+r}}{\left|X_{t}-Y_{t}\right|_{\mathbb{H}}^{\delta(2+r)}} d t \leq|x-y|_{\mathbb{H}}^{2 \delta}
$$

By Hölder's inequality, we have

$$
\begin{aligned}
\int_{0}^{T} \frac{\eta_{t}^{2}\left|X_{t}-Y_{t}\right|_{\sigma_{t}}^{2}}{\left|X_{t}-Y_{t}\right|_{\mathrm{H}}^{2 \delta}} d t & \leq\left(\int_{0}^{T} \frac{\eta_{t}^{2}\left|X_{t}-Y_{t}\right|_{\sigma_{t}}^{2+r}}{\left|X_{t}-Y_{t}\right|_{\mathbb{H}}^{\delta(2+r)}} d t\right)^{\frac{2}{2+r}}\left(\int_{0}^{T} \eta_{t}^{2} d t\right)^{\frac{r}{2+r}} \\
& \leq\left(\frac{\vartheta_{T}^{2}}{\delta \gamma}|x-y|_{\mathbb{H}}^{2 \delta}\right)^{\frac{2}{2+r}} \cdot \vartheta_{T}^{\frac{2 r}{2+r}}\left(\int_{0}^{T} \zeta_{t}^{2} \mathrm{e}^{-\delta \omega t} d t\right)^{\frac{r}{2+r}}
\end{aligned}
$$

Note that

$$
\vartheta_{T}^{2}=\frac{4 \delta^{-2}|x-y|_{\mathbb{H}}^{2 \delta}}{\left(\int_{0}^{T} \zeta_{t} \mathrm{e}^{-\delta \omega t} d t\right)^{2}}
$$

we obtain

$$
\int_{0}^{T} \frac{\eta_{t}^{2}\left|X_{t}-Y_{t}\right|_{\sigma_{t}}^{2}}{\left|X_{t}-Y_{t}\right|_{\mathbb{H}}^{2 \delta}} d t \leq 4 \delta^{-\frac{2(3+r)}{2+r}} \gamma^{-\frac{2}{2+r}} \frac{\left(\int_{0}^{T} \zeta_{t}^{2} \mathrm{e}^{-\delta \omega t} d t\right)^{\frac{r}{2+r}}}{\left(\int_{0}^{T} \zeta_{t} \mathrm{e}^{-\delta \omega t} d t\right)^{2}}|x-y|_{\mathbb{H}}^{\frac{2(4+r-q)}{2+r}}
$$


Now it is clear that (30) holds.

(5) Estimate of $\mathbb{E} R_{T}^{\alpha /(\alpha-1)}$.

Denote $\beta=\alpha /(\alpha-1)$. Since $R_{t}$ is a $\mathbb{P}$-martingale, for any $p, q>1$ with $q=p /(p-1)$, we have

$$
\begin{aligned}
& \mathbb{E}_{\mathbb{P}} R_{T}^{\alpha /(\alpha-1)} \\
& =\mathbb{E}_{\mathbb{P}} \exp \left(\beta N_{T}-\frac{1}{2} p \beta^{2}[N]_{T}\right) \exp \left(\frac{1}{2} \beta(\beta p-1)[N]_{T}\right) \\
& \leq\left[\mathbb{E}_{\mathbb{P}} \exp \left(p \beta N_{T}-\frac{1}{2} p^{2} \beta^{2}[N]_{T}\right)\right]^{1 / p} \cdot\left[\mathbb{E}_{\mathbb{P}} \exp \left(\frac{1}{2} \beta q(\beta p-1)[N]_{T}\right)\right]^{1 / q} \\
& =\left[\mathbb{E}_{\mathbb{P}} \exp \left(\frac{1}{2} \beta q(\beta p-1)[N]_{T}\right)\right]^{1 / q} \text {. }
\end{aligned}
$$

Hence we get (23) by using the estimate (40) and letting $p$ go to 1 .

(6) Suppose that $\sigma$ is independent of $(t, \omega)$. And we take $\zeta_{t}$ in (22) as a constant $\zeta$. Then we can simplify $\Theta_{T}$ as follows.

$$
\begin{aligned}
\Theta_{T} & =4 \delta^{-\frac{2(3+r)}{2+r}} \gamma^{-\frac{2}{2+r}} \frac{\left(\zeta^{2} \int_{0}^{T} \mathrm{e}^{-\delta \omega t} d t\right)^{\frac{r}{2+r}}}{\left(\zeta \int_{0}^{T} \mathrm{e}^{-\delta \omega t} d t\right)^{2}} \\
& =4 \delta^{-\frac{2(3+r)}{2+r}} \gamma^{-\frac{2}{2+r}} \zeta^{-\frac{4}{2+r}}\left[(\delta \omega)^{-1}\left(1-\mathrm{e}^{-\delta \omega T}\right)\right]^{-\frac{4+r}{2+r}} \\
& =4 \delta^{-1} \gamma^{-\frac{2}{2+r}} \zeta^{-\frac{4}{2+r}}\left[\omega^{-1}\left(1-\mathrm{e}^{-\delta \omega T}\right)\right]^{-\frac{4+r}{2+r}}=\widetilde{\Theta}_{T}
\end{aligned}
$$

Remark 4.2. We refer to [28, Corollary 1.3] for sufficient conditions for (22).

Remark 4.3. Consider the following multivalued stochastic differential equation on $\mathbb{R}^{d}$

$$
d X_{t}+A X_{t} d t \ni b\left(X_{t}\right) d t+d W_{t}, \quad X_{0}=x \in \overline{D(A)},
$$

where $A$ is a maximal monotone operator on $\mathbb{R}^{d}$ with $D(A)^{\circ} \neq \emptyset$, Wt is a Wiener process on a filtered probability space $\left(\Omega, \mathscr{F},\left(\mathscr{F}_{t}\right)_{t \geq 0}, \mathbb{P}\right), b: \mathbb{R}^{d} \rightarrow \mathbb{R}^{d}$ is a continuous function such that there exists some $\omega \in \mathbb{R}$

$$
\langle x-y, b(x)-b(y)\rangle \leq \omega|x-y|^{2}, \quad x, y \in \mathbb{R}^{d} .
$$

Then by [23, Thorem 2.8], the solution $X_{t}$ exists. For the transition semigroup $P_{t}$ associated with $X_{t}$, we have

$$
\left(P_{t} f\right)^{\alpha}(x) \leq \exp \left(\frac{\alpha \omega|x-y|^{2}}{(\alpha-1)\left(1-\mathrm{e}^{-2 \omega t}\right)}\right) P_{t} f^{\alpha}(y) .
$$

for every $t>0, \alpha>1, x, y \in \overline{D(A)}$ and $f \in \mathscr{C}_{b}^{+}(\overline{D(A)})$. 
The proof is similar to the proof of Theorem 4.1 except that we choose another simpler drift. That is, instead of (27), we consider the following coupled equations

$$
\left\{\begin{array}{l}
d X_{t}+A X_{t} d t \ni d W_{t}+b\left(X_{t}\right) d t-\xi_{t}|x-y| \frac{X_{t}-Y_{t}}{\left|X_{t}-Y_{t}\right|} \mathbb{1}_{\{t<\tau\}} d t \\
d Y_{t}+A Y_{t} d t \ni d W_{t}+b\left(Y_{t}\right) d t
\end{array}\right.
$$

with initial data $X_{0}=x$ and $Y_{0}=y$, where $\tau$ is the coupling time of $X_{t}$ and $Y_{t}$ and

$$
\xi_{t}=\frac{\mathrm{e}^{-\omega t}}{\int_{0}^{T} \mathrm{e}^{-2 \omega s} d s}, \quad t \geq 0
$$

\section{Applications}

Zhang [31, Corollary 5.3] studied Feller property of $P_{t}$. We prove strong Feller property for $P_{t}$ under additional conditions.

Theorem 5.1. Assume (H1)-(H5), $\left(H 6^{\prime}\right)$ and (22) with $q<4+r$. Then the semigroup $P_{t}$ is strongly Feller and the following estimate holds

$$
\left|P_{t} f(x)-P_{t} f(y)\right| \leq\|f\|_{\infty} \Theta_{t}^{1 / 2}|x-y|_{\mathbb{H}}^{\frac{4+r-q}{2+r}} \cdot \exp \left(\frac{1}{2} \Theta_{t}|x-y|_{\mathbb{H}}^{\frac{2(4+r-q)}{2+r}}\right)
$$

for every $t>0, x, y \in \overline{D(A)}$ and $f \in \mathscr{B}_{b}(\overline{D(A)})$. Moreover, if the invariant measure $\mu$ exist, then for every $f$ in $L^{p}(\overline{D(A)}, \mu)$ with $p>1, P_{t} f$ is continuous on $\overline{D(A)}$.

Proof. The last statement follows directly from the Harnack inequality (23) by applying [12, Proposition 4.1]. We only need to show the estimate (44).

We follow the notation in the proof of Theorem 4.1. By (31), we see

$$
\begin{aligned}
\left|P_{T} f(x)-P_{T} f(y)\right| & =\left|\mathbb{E}_{\mathbb{Q}} f\left(X_{T}\right)-\mathbb{E}_{\mathbb{P}} f\left(Y_{T}\right)\right|=\left|\mathbb{E}_{\mathbb{P}} R_{T} f\left(X_{T}\right)-\mathbb{E}_{\mathbb{P}} f\left(X_{T}\right)\right| \\
& =\mathbb{E}_{\mathbb{P}}\left|f\left(X_{T}\right)\left(1-R_{T}\right)\right| \leq\|f\|_{\infty} \mathbb{E}_{\mathbb{P}}\left|1-R_{T}\right| .
\end{aligned}
$$

It is clear

$$
\left(\mathbb{E}_{\mathbb{P}}\left|1-R_{T}\right|\right)^{2} \leq \mathbb{E}_{\mathbb{P}}\left(1-R_{T}\right)^{2}=\mathbb{E}_{\mathbb{P}} R_{T}^{2}-1
$$

By (41), we know

$$
\mathbb{E}_{\mathbb{P}} R_{T}^{2} \leq \exp \left(\Theta_{T}|x-y|_{\mathbb{H}}^{\frac{2(4+r-q)}{2+r}}\right)
$$

Using the elementary inequality $\mathrm{e}^{r}-1 \leq r \mathrm{e}^{r}, r \geq 0$, we can deduce from (46) and (47) to get

$$
\left(\mathbb{E}_{\mathbb{P}}\left|1-R_{T}\right|\right)^{2} \leq \Theta_{T}|x-y|_{\mathbb{H}}^{\frac{2(4+r-q)}{2+r}} \exp \left(\Theta_{T}|x-y|_{\mathbb{H}}^{\frac{2(4+r-q)}{2+r}}\right)
$$

Substitute the estimate above into (45) we can obtain (44). 
Theorem 5.2. Assume (H1)-(H5), (H6 $\left.6^{\prime}\right),(22), \sigma$ is independent of $(t, \omega)$ and the invariant measure $\mu$ of the semigroup $P_{t}$ exist. Then

1. The invariant measure $\mu$ is fully supported on $\overline{D(A)}$.

2. For every $x \in \overline{D(A)}, t>0$, the transition density $p_{t}(x, \cdot)$ (with respect to $\mu$ ) exist and for every $\alpha>1$

$$
\left\|p_{t}(x, \cdot)\right\|_{L^{\alpha}(\overline{D(A)}, \mu)} \leq\left[\int_{\overline{D(A)}} \exp \left(-\frac{\alpha}{2} \widetilde{\Theta}_{t}|x-y|_{\mathbb{H}^{\frac{2(4+r-q)}{2+r}}}\right) \mu(d y)\right]^{-(\alpha-1) / \alpha} .
$$

3. Suppose $K \leq 0$.

(a) If $q=2$ and $\lambda \omega<\gamma$, where $\lambda$ is the constant such that $|\cdot|_{\mathbb{H}} \leq \lambda|\cdot|_{\mathbb{V}}$, then $P_{t}$ is hyperbounded.

(b) If $q>2$, then $P_{t}$ is ultrabounded. More precisely, there exist some constant $c>0$ such that

$$
\left\|P_{t}\right\|_{2 \rightarrow \infty} \leq \exp \left(c\left(1+t^{-\frac{q}{q-2}}\right)\right) .
$$

Consequently, $P_{t}$ is compact for large $t>0$ for both cases.

Proof. (1) If supp $\mu \neq \overline{D(A)}$, then there exists some $x_{0} \in \overline{D(A)}$ and $r>0$ such that $\mu\left(B_{r}\left(x_{0}\right)\right)=0$, where $B_{r}\left(x_{0}\right)=\left\{y \in \overline{D(A)}:\left|y-x_{0}\right| \leq r\right\}$.

Applying (23) to the function $\mathbb{1}_{B_{r}\left(x_{0}\right)}$ for $\alpha=2$ and $t \geq 0$, we have

$$
\left(P_{t} \mathbb{1}_{B_{r}\left(x_{0}\right)}\right)^{2}(x) \exp \left(-\widetilde{\Theta}_{t}|x-y|_{\mathbb{H}}^{\frac{2(4+r-q)}{2+r}}\right) \leq P_{t} \mathbb{1}_{B_{r}\left(x_{0}\right)}(y) .
$$

Hence, by integrating both sides of (49) with respect to $\mu(d y)$, we obtain

$$
\begin{aligned}
& \left(P_{t} \mathbb{1}_{B_{r}\left(x_{0}\right)}\right)^{2}(x) \int_{\overline{D(A)}} \exp \left(-\tilde{\Theta}_{t}|x-y|_{\mathbb{H}}^{\frac{2(4+r-q)}{2+r}}\right) \mu(d y) \\
\leq & \mu\left(P_{t} \mathbb{1}_{B_{r}\left(x_{0}\right)}\right)=\mu\left(\mathbb{1}_{B_{r}\left(x_{0}\right)}\right)=0 .
\end{aligned}
$$

This implies $P_{t}\left(x_{0}, B_{r}\left(x_{0}\right)\right)=0$ for all $t \geq 0$. Therefore,

$$
\mathbb{P}\left(\left|X_{t}\left(x_{0}\right)-x_{0}\right|_{\mathbb{H}} \leq r\right)=0, \quad t>0,
$$

where $X_{t}\left(x_{0}\right)$ denotes the solution to (1) with $X_{0}\left(x_{0}\right)=x_{0}$.

Since $X_{t}$ is continuous on $\mathbb{H}$, by letting $t \rightarrow 0$ in (50), we have

$$
\mathbb{P}\left(\left|X_{0}\left(x_{0}\right)-x_{0}\right|_{\mathbb{H}} \leq r\right)=0 .
$$

But obviously this is impossible. So it must be supp $\mu=\overline{D(A)}$.

(2) We refer to [21] or [28] for the idea of the proof.

(3) Since $K \leq 0$, for any $t>0$, we know

$$
\frac{K}{1-\mathrm{e}^{-\delta K t}} \leq \frac{1}{\delta t}
$$


Therefore, by Theorem 4.1, there exist some constant $C_{8}$ such that for every $x, y \in \overline{D(A)}$ and $t>0$,

$$
\left(P_{t} f\right)^{2}(x) \exp \left(-\frac{C_{8}|x-y|_{\mathbb{H}}^{\frac{2(4+r-q)}{2+r}}}{t^{\frac{4+r}{2+r}}}\right) \leq P_{t} f^{2}(y)
$$

where $f \in L^{2}(\overline{D(A)}, \mu)$ with $\mu\left(f^{2}\right)=1$.

By integrating both sides of (51) with respect to $\mu(d y)$ over $B_{1}(0)=\left\{x \in \overline{D(A)}:|x|_{\mathbb{H}} \leq\right.$ $1\}$, we see for every $x \in \overline{D(A)}$ and $t>0$,

$$
\left(P_{t} f\right)^{2}(x) \leq \frac{1}{\mu\left(B_{1}(0)\right)} \exp \left(\frac{C_{8}\left(1+|x|_{\mathbb{H}}\right)^{\frac{2(4+r-q)}{2+r}}}{t^{\frac{4+r}{2+r}}}\right) .
$$

(i) If $q=2$, then by taking square and integration with respect to $\mu(d x)$ for both sides of the equation (52), and using Theorem 3.1, we have

$$
\int_{\overline{D(A)}}\left(P_{t} f\right)^{4}(x) \mu(d x) \leq \frac{1}{\mu\left(B_{1}(0)\right)} \int_{\mathbb{H}} \exp \left(\frac{C_{8}\left(1+|x|_{\mathbb{H}}\right)^{2}}{t^{\frac{4+r}{2+r}}}\right) \mu(d x)<\infty
$$

for $t>0$ big enough. This proves $\left\|P_{t}\right\|_{2 \rightarrow 4}<\infty$ for sufficiently big $t>0$. That is, $P_{t}$ is hyperbounded.

(ii) Assume $q>2$. Then the inequality (15) implies

$$
d \mathrm{e}^{\theta\left|X_{t}\right|_{\mathrm{H}}^{q}} \leq\left(C_{9}-\gamma^{\prime \prime \prime}\left|X_{t}\right|_{\mathrm{H}}^{2(q-1)} \mathrm{e}^{\theta\left|X_{t}\right|_{\mathrm{H}}^{q}}\right) d t+d M_{t}
$$

for some constant $C_{9}, \theta, \gamma^{\prime \prime \prime}>0$.

Let $g(t)$ be the solution to the following equation

$$
d g(t)=\left(C_{9}-\gamma^{\prime \prime \prime} \theta^{-\frac{2(q-1)}{q}} g(t)[\log g(t)]^{\frac{2(q-1)}{q}}\right) d t
$$

with $g(0)=\mathrm{e}^{\theta|x|_{\mathrm{H}}^{q}}$.

By the comparison theorem, we have

$$
\mathbb{E} \mathrm{e}^{\theta\left|X_{t}(x)\right|_{\mathrm{H}}^{q}} \leq g(t) \leq \exp \left(C_{9}\left(1+t^{-\frac{q}{q-2}}\right)\right)
$$

for some constant $C_{9}>0$. By inequality (52) we have

$$
\left\|P_{t} f\right\|_{\infty}=\left\|P_{t / 2} P_{t / 2} f\right\|_{\infty} \leq C_{10} \sup _{x \in \overline{D(A)}} \mathbb{E} \exp \left[\frac{C_{11}}{t^{\frac{4+r}{2+r}}}\left|X_{t / 2}(x)\right|_{\mathbb{H}}^{\frac{2(4+r-q)}{2+r}}\right]
$$

for some constants $C_{10}, C_{11}>0$.

By using Young's inequality, we see

$$
C_{10} \sup _{x \in \overline{D(A)}} \mathbb{E} \exp \left[\frac{C_{11}}{t^{\frac{4+r}{2+r}}}\left|X_{t / 2}(x)\right|_{\mathbb{H}}^{\frac{2(4+r-q)}{2+r}}\right] \leq \varepsilon \cdot\left|X_{t / 2}^{x}\right|_{\mathbb{H}}^{q}+\varepsilon^{\prime} t^{-\frac{q}{q-2}}
$$


for arbitrary $\varepsilon>0$. By considering small enough $\varepsilon>0$, it follows from the inequality (56) above and (54) and (55) we can obtain (48). This proves that $P_{t}$ is ultrabounded.

Since $P_{t}$ has transition density with respect to $\mu$, we know $P_{t}$ is compact in $L^{2}(\overline{D(A)}, \mu)$ for large $t>0$ for these two cases $(q=2$ or $q>2)$ by [14, Lemma 3.1].

By [29, Section 2], we can get some heat kernel inequalities from Harnack inequality. Especially we have the following log-Harnack inequality (57) and an entropy-cost type inequality (58).

Theorem 5.3. Assume (H1)-(H5), $\left(H 6^{\prime}\right)$ and (22) with $q<4+r$. Then

1. For every $T>0, x, y \in \overline{D(A)}, f \geq 1$, and $f \in \mathscr{B}_{b}(\overline{D(A)})$

$$
P_{T}(\log f)(x) \leq \log P_{T} f(y)+\frac{1}{2} \Theta_{T}|x-y|_{\mathbb{H}}^{\frac{2(4+r-q)}{2+r}} .
$$

2. For every $T>0, f \in \mathscr{B}_{b}^{+}(\overline{D(A)})$ such that $\mu(f)=1$,

$$
\mu\left(P_{T}^{*} f \log \left(P_{T}^{*} f\right)\right) \leq \frac{\Theta_{T}}{2} \inf _{\pi \in \mathscr{C}(\mu, f \mu)} \int_{\overline{D(A)}} \int_{\overline{D(A)}}|x-y|_{\mathrm{H}}^{\frac{2(4+r-q)}{2+r}} d \pi,
$$

where $\mathscr{C}(\mu, f \mu)$ denotes all the coupling of $\mu$ and $f \mu$.

Proof. Inequality (57) follows as [29, Proposition 2.2]. Integrating both sides of (57) with respect to $\pi \in \mathscr{C}(\mu, f \mu)$, and minimizing in $\pi$, we get

$$
\mu\left(\left(P_{t}^{*} f\right) \log P_{t}^{*} f\right) \leq \mu\left(\log \left(P_{t} P_{t}^{*} f\right)\right)+\frac{\Theta_{T}}{2} W_{2}(\mu, f \mu)^{2} .
$$

Now we obtain (58) by noting

$$
\mu\left(\log \left(P_{t} P_{t}^{*} f\right)\right) \leq \log \mu\left(P_{t} P_{t}^{*} f\right)=\log 1=0
$$

due to the fact that $\mu$ is invariant for $P_{t}$ and $P_{t}^{*}$.

\section{Acknowledgment}

This work was supported in part by the DFG through the International Graduate College "Stochastics and Real World Models". The author would like to thank the encouragement of Professors Michael Röckner and Feng-Yu Wang.

\section{References}

[1] S. Aida, H. Kawabi, Short time asymptotics of a certain infinite dimensional diffusion process, in: L. Desreusefond, B. Øksendal, A. S. Üstünel (Eds.), Stochastic analysis and related topics, VII (Kusadasi, 1998), Vol. 48 of Progr. Probab., Birkhäuser Boston, Boston, MA, 2001, pp. 77-124. 
[2] S. Aida, T. Zhang, On the small time asymptotics of diffusion processes on path groups, Potential Anal. 16 (1) (2002) 67-78.

[3] M. Arnaudon, A. Thalmaier, F.-Y. Wang, Harnack inequality and heat kernel estimates on manifolds with curvature unbounded below, Bull. Sci. Math. 130 (3) (2006) 223-233.

[4] D. Bakry, M. Ledoux, Z. Qian, Logarithmic Sobolev inequalities, Poincaré inequalities and heat kernel bounds, unpublished (1997).

[5] A. Bensoussan, A. Rascanu, Stochastic variational inequalities in infinite-dimensional spaces, Numer. Funct. Anal. Optim. 18 (1-2) (1997) 19-54.

[6] S.G. Bobkov, I. Gentil, M. Ledoux, Hypercontractivity of Hamilton-Jacobi equations, J. Math. Pures Appl. (9) 80 (7) (2001) 669-696.

[7] H. Brézis, Opérateurs maximaux monotones et semi-groupes de contractions dans les espaces de Hilbert, North-Holland Publishing Co., Amsterdam, 1973, north-Holland Mathematics Studies, No. 5. Notas de Matemática (50).

[8] E. Cépa, Équations différentielles stochastiques multivoques, C. R. Acad. Sci. Paris Sér. I Math. 319 (10) (1994) 1075-1078.

[9] E. Cépa, Équations différentielles stochastiques multivoques, in: Séminaire de Probabilités, XXIX, Vol. 1613 of Lecture Notes in Mathematics, Springer, Berlin, 1995, pp. 86-107.

[10] E. Cépa, Problème de Skorohod multivoque, Ann. Probab. 26 (2) (1998) 500-532.

[11] E. Cépa, D. Lépingle, Diffusing particles with electrostatic repulsion, Probab. Theory Related Fields 107 (4) (1997) 429-449.

[12] G. Da Prato, M. Röckner, F.-Y. Wang, Singular stochastic equations on hilbert spaces: Harnack inequalities for their transition semigroups, J. Funct. Anal. In Press.

[13] F.-Z. Gong, F.-Y. Wang, Heat kernel estimates with application to compactness of manifolds, Q. J. Math. 52 (2) (2001) 171-180.

[14] F.-Z. Gong, F.-Y. Wang, Functional inequalities for uniformly integrable semigroups and application to essential spectrums, Forum Math. 14 (2) (2002) 293-313.

[15] H. Kawabi, Functional inequalities and an application for parabolic stochastic partial differential equations containing rotation, Bull. Sci. Math. 128 (8) (2004) 687-725.

[16] H. Kawabi, The parabolic Harnack inequality for the time dependent GinzburgLandau type SPDE and its application, Potential Anal. 22 (1) (2005) 61-84.

[17] P. Krée, Diffusion equation for multivalued stochastic differential equations, J. Funct. Anal. 49 (1) (1982) 73-90. 
[18] N.V. Krylov, B. L. Rozovskii, Stochastic evolution equations, in: Stochastic differential equations: theory and applications, Vol. 2 of Interdiscip. Math. Sci., World Sci. Publ., Hackensack, NJ, 2007, pp. 1-69.

[19] S.-X. Ouyang, Harnack inequalities and applications for stochastic equations, Ph.D. thesis, Bielefeld University, 2009, available on http://bieson.ub.uni-bielefeld.de/volltexte/2009/1463/pdf/ouyang.pdf.

[20] S.-X. Ouyang, M. Röckner, F.-Y. Wang, Harnack Inequalities and Applications for Ornstein-Uhlenbeck Semigroups with Jump, 2009, Preprint.

[21] M. Röckner, F.-Y. Wang, Harnack and functional inequalities for generalized Mehler semigroups, J. Funct. Anal. 203 (1) (2003) 237-261.

[22] M. Röckner, F.-Y. Wang, Supercontractivity and ultracontractivity for (nonsymmetric) diffusion semigroups on manifolds, Forum Math. 15 (6) (2003) 893-921.

[23] J. Ren, J. Wu, and X. Zhang, Exponential ergodicity of non-lipschitz multivalued stochastic differential equations, to appear in Bull. Sci. math. (2009).

[24] F.-Y. Wang, Logarithmic Sobolev inequalities on noncompact Riemannian manifolds, Probab. Theory Related Fields 109 (3) (1997) 417-424.

[25] F.-Y. Wang, Harnack inequalities for log-Sobolev functions and estimates of logSobolev constants, Ann. Prob. 27 (2) (1999) 653-663.

[26] F.-Y. Wang, Logarithmic Sobolev inequalities: conditions and counterexamples, J. Operator Theory 46 (1) (2001) 183-197.

[27] F.-Y. Wang, Dimension-free Harnack inequality and its applications, Front. Math. China 1 (1) (2006) 53-72.

[28] F.-Y. Wang, Harnack inequality and applications for stochastic generalized porous media equations, Ann. Probab. 35 (4) (2007) 1333-1350.

[29] F.-Y. Wang, Heat kernel inequalities for convexity of manifold and curvature condition, preprint, 2009.

[30] E. Zeidler, Nonlinear functional analysis and its applications. II/A, Springer-Verlag, New York, 1990, linear monotone operators, Translated from the German by the author and Leo F. Boron.

[31] X. Zhang, Skorohod problem and multivalued stochastic evolution equations in Banach spaces, Bull. Sci. Math. 131 (2) (2007) 175-217. 